\title{
Die zwei provenzalischen Gedichte, das Glaubens- und das Beichtsbekenntnis der Pariser Hs, fonds lat. 11312.
}

Im Grundrifs der provenzalischen Litteratur \$ I I bezeichnet Bartsch die von P. Meyer in seinen Anciennes Poésies réligieuses aus Hs. suppl. lat. I 743 (jetzt fonds lat. I I 3 I 2) der Pariser Nationalbibliothek Bl. I $22 \mathbf{v}^{0} \mathrm{ff}$. veröffentlichten Gedichte (ein Glaubens- und ein Beichtbekenntnis) als ,in Versen von ungleichem Mafse“ gedichtet. Hiermit wird aber das wirkliche Versmals nicht deutlich genug festgestellt. Meyer drückt sich in seiner Vorbemerkung zwar präziser aus, seine Worte decken sich jedoch nicht völlig mit der metrischen Konstruktion seiner Ausgabe (die Hs. überliefert den Text wie Prosa und setzt nur nach den Strophen schliefsenden ent aber auch hier nicht konsequent Punkte) und auch nicht mit dem metrischen Gebilde, wie es nach meiner Ansicht aus des Dichters Hand hervorging. Meyer sagt: „Ce sont deux hymnes (ou peut-être un seul en deux parties); le premier commence par un acte de foi et finit par une prière: c'est un récit vif et animé des principales circonstances de la vie et de la passion du Christ; le second est une sorte d'acte de contrition un peu vague et un peu long. Du reste il faut reconnaître dans celle piece (ou ces pièces) une grande habileté de versification. Sauf les quatre premiers vers de douze pieds qui sont comme la préface du poëme, ce sont de petits vers disposés par strophes de trois vers dont le dernier est constamment un en t. Ces strophes sont de deux ispeces: l'une de trois vers de six syllabes, l'autre de deux vers de quatre et d'un de six ou parfois huit syllabes, ces strophes n'étant point alternées, mais disposées par séries de plusieurs strophes de même nature. Celle en vers inégaux a un rhythme léger et sautillant d'un effet assez joli, mais que vient heureusement remplacer, dans les endroits où le ton du récit s'élève, le rhythme plus grave de la strophe en vers de six syllabes."

Meyer spricht dann noch über die Verwendung einer ähnlichen Strophenform bei Giraud de Cabreira und Giraud de Calanson. Über diese Schweifreimstrophe hat seitdem ausführlich Suchier in der Einleitung zur Reimpredigt: Grant mal fist Adam gehandelt. Sonst ist mir nicht bekannt, dafs über den Bau unseres Gedichtes eine weitere Äufserung gethan wäre, abgesehen von der Wiedergabe des Meyer'schen Textes von $\amalg$ Str. $I-27$ in Bartsch's Chrestomathie prov. ${ }^{4}$ Sp. $19 \mathrm{ff}$. 
Da ich den Text mir vor einigen Jahren von neuem abgeschrieben hatte, in der Absicht eine meiner französischen analoge Sammlung der ältesten provenzalischen Sprachdenkmäler zu veranstalten, zu derselben aber noch nicht sobald kommen werde, gebe ich hier den Text nach meiner den Meyer'schen Abdruck hier und da berichtigenden Abschrift, jedoch gleich in der metrischen Form, die ich für die richtige halte und mit den dadurch bedingten Besserungen. In sprachlicher Beziehung lasse ich den Text so ziemlich unangetastet, da in eine sprachliche Untersuchung desselben einzutreten, zur Zeit aufser meiner Absicht liegt. Ich schicke noch einige das Metrum klarstellende und die eingeführten Änderungen rechtfertigende Bemerkungen vorauf.

I.

Betrachten wir zunächst das erste Gedicht oder Zeile I-I I 3. Wir haben in ihm lauter zweireimige Strophen aus drei Zeilen anzunehmen. Der zweite Reim begegnet immer in der letzten Zeile und geht durch das ganze Gedicht (sogar auch durch das zweite Gedicht) hindurch auf ent aus.

Der erste Reim verknüpft die zwei ersten Zeilen und wechselt von Strophe zu Strophe, nur zufällig kehrt derselbe Rein in mehreren aufeinander folgenden wieder. Der Reim ist meist männlich. An Stelle des Reimes begegnen einige Male Assonanzen.

Die dritte die Strophe abschliefsende Verszeile ist durchweg ein Sechsilbner, die ersten beiden sind meist auch Sechssilbner, in Strophe $\mathrm{I}-4$ sind es jedoch Dreisilbner. Sonst haben wir noch Strophe $8-14,20-25$ und $27-28$ in Zeile 1,2 Viersilbner. Vielleicht ist nun die entbehrliche Strophe 26 erst später eingeschoben und liefsen sich durch ihre Ausscheidung 2 Absätze von 4. und 6. Silbnerstrophen herstellen, zwischen welche 5 reine Sechsilbnerstrophen geschoben wären, ebenso wie ihnen 3 solche voraufgehen und 12 nachfolgen. Dieser Auffassung des metrischen Gebildes stehen nur folgende Bedenken entgegen.

I. Die Strophen $\mathbf{I}-5$ scheinen nur 5 einfache Langzeilen $z u$ scin. Es sind jedoch nur geringfügige Änderungen erforderlich um dreizeilige Strophen herzustellen. Strophe 5, welche Meyer ohne das handschriftliche Verweisungszeichen zu beachten, als Eingangszeile druckte, darf man schwerlich als vierzeilige Strophe auffassen, deren erste drei Verse auf a reimten, da Reime auf unbetonten Vokal unseren Dichter fremd sind. 2. Strophe $15-17$ bilden der Überlieferung nach nur 2 Strophen von 4 und 5 Zeilen. Die vorgenommene Änderung dürfte auf keinen Widerspruch stofsen. - 3. Meyer lälst für die Schlufszeile der Strophen auch den Achtsilbner zu. Die Überlieferung zeigt aber nur vier solcher Strophen, nämlich a) 2 I bis 23. Die beiden ersten Zeilen sind hier Viersilbner, da nun unmittelbar vorher I und unmittelbar nachher 2 regelrechte Vierund Sechssilbnerstrophen gehen, so wird man diese einzigen drei Achtsilbner in Sechssilbner kürzen dürfen, was, wie mein Text zeigt, leicht möglich ist. b) Strophe 33, wo sicher eine analoge Kürzung 
vorzunehmen ist. - 4. Zwischen Strophe 38 und 39 steht eine überschüssig Zeile. Ich betrachte sie als Interpolation, da sie völlig überllüssig erscheint.

II.

Ich wende mich zum zweiten Gedicht. Dieses scheint mir durchaus in dreizeiligen Sechssilbnerstrophen abgefafst, allerdings mufs zugegeben werden, dafs aufser einigen Textkorruptionen, auch offenbar eine Anzahl beabsichtigter Änderungen von dem Redaktor des erhaltenen Textes vorgenommen sind, wodurch neue Strophenformen und darunter eine, welche den Reim noch mehr ins Ohr fallen liefs, entstanden. Das Bestreben nach gröfserem Reimreichtum bekundete sich schon bei dem ursprünglichen Dichter, durch öftere Einführung des Binnenreims. Im ersten Gedicht zeigt sich dieser nur einmal in der dritten Strophenzeile (Str. 19), im vorliegenden tritt er viel häufiger auf (vgl. Ib, 2a, 6a, 10b, 15a, 21a, 24a, 27a, b) und der Überarbeiter hat sich $27 \mathrm{a}$ b verleiten lassen, ihn noch durch eine Reihe, allerdings nichtssagender Zusätze zu vermehren. (Hier wie im Beginn des Gedichts hat Meyer das strophische Gebilde völlig verkannt. $\mathrm{Zu}$ Ic hat er irrigerweise die erste Hälfte von 2a und demnach die zweite Hälfte zu $2 c$ gezogen). Sonst hat er die Strophe noch umgebildet zu einer aus 3 Achtsilbnern (5) aus 2 Vicr- und einem Sechssilbner $(6,7,23)$ aus 2 Sieben- und I Scchssilbner (43), aus 2 Sechs- und I Achtsidbner (42), mit Unterdrückung einer Zeile zu einer aus 2 Drei- und I Sechssilbner (10) und mit Hinzufägung einer Zeile aus 4 Sechssilbnern (19). Jedenfalls fehlerhafte Einzelverse sind: ( $1 \mathrm{a}, \mathrm{c}, 28 \mathrm{a}, \mathrm{b}, 29 \mathrm{a}, 30 \mathrm{c}, 32 \mathrm{a}, 36 \mathrm{~b}$ (falscher Reimvokal), 36c.

I.

I Damrideu

Aor eu,

Lo pair'omnipotent,

I

2 Qui creet

Terra e cel

F quant es de neient. 2

3 E aur

So fil bo

Jesu Christ esament

4 Qui naissia

De Maria

Per nostre salvament.

3
5 Sia ela beneeita

Que de lui fo eseinta

Ta gloriosament,

6 Nol coseub a deleit, 5

$\mathrm{Ni}$ ac dolor a leit, 6

$\mathrm{Ni}$ sos cors naframent; 7

7 A la nativitat 8

De sa virginitat 9

Per lui no pert neent. 10

8 Aquest cre eu I I

Omenadeu, $\quad 12$

Lui en tra a garent; 13

I ab Eu aor damrideu.

2b Cel e terra.

3 b So bo fil.

3c ih'u von Meyer, wie fast allgemein, fälschlich Jhesu aufgelöst, zu esament vgl. I $\mathrm{r} 29 \mathrm{c}$. $4 \mathrm{a}$ nasquet. $\quad 5 \mathrm{a}-\mathrm{c}$ Vom Kopisten am oberen Rande nachgetragen, aber durch Verweisungszeichen als nach 4c gehörig kenntlich gemacht. 5a Ela sia 5b esenta. sid. 6b Meyer: Ni n'ac. 7b Meyer: virginat; $7 \mathrm{c}$ Meyer: part. 
9 Qu'el me plasmet I4 E m'aspiret 15

Em reems humilment. I6

Io Quant me compret, I7 No m'acaptet 18

De fin aur ni d'argent, 19

I Qu'ans donet se 20

Non autra re 2 I

Per ma vida garent. $\quad 22$

12 D'u de sos druxtz 23

El fo vendutz 24

Trenta diners d'argent. 25

13 Nol desfiet, 26

Quant lo baiet, 27

Cum veng al traïment. $\quad 28$

14 Receub l'aver 29

Ret Deu lo ver 30

A la malvasa gent. 3 I

I5 Donc fo pres e liatz 32

E cum laire jutgatz 34

A mor e a torment, 35

16 E aisi despolatz $\quad 36$

Cum de maire fo natz, 37

$\mathrm{E}$ batutz malament.

I7 E per nostres pecatz 38

E la cros fo levatz 39

On el fetz plaguament 40

I 8 De sos omes a Deu 41

- D'aquels, sel plaz, si'eu

C'aiso cre fermament -. 43

19 Clavellero el fust 44

Las mas de l'ome just 45

Es pes apres fortment. 46

20 Nos rancuret,

Qu'ans perdonet

\section{8}

A la malvasa gent.

49

2 I Prega sa jus

Lo paire sus

$5 \mathrm{I}$

Fasa perdonament.

4
5
6
7
8
9
1
2
3
4
5
6
7
8
9
30

22 Mostral bos om,
Que aital fasa om
D'autrui malmenament.
$23 \mathrm{Si}$ con ac set $\quad 56$
Fel e azet 57

$\mathrm{Li}$ mesquero la gent. $\quad 5^{8}$

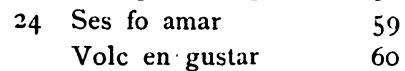

Per nostre salvament; 6 I

$25 \mathrm{E}$ e derrer 62

D'un cavaler 63

Fo plagatz mortalment. 64

26 (Cant la lansal ferit 65

Sanc e aiga'n isit 66

Tot aveüdament.) $\quad 67$

27 So fo lo prest 68

Que fo proferts 69

Pel meu delivrament; 70

28 E el fo morst $7 \mathbf{I}$

Per los meus tortst 72

E mes el monument. $\quad 73$

29 Cum paubres om fo morz 74

E resor cum Deus forz 75

Al ters jorn, verament. 76

30 Pois als seus aparec, 77

$\mathrm{Ab}$ els manget e bec 78

E conortet los gens. 79

$\begin{array}{lll}42 & 31 & \mathrm{E} \\ & \text { poiet en el cel } & 80\end{array}$

$\mathrm{O}$ estau sei fiel $8 \mathrm{I}$

Tro ven'al jutjament, $\quad 82$

52 Que metra bos en gloria 83

El mal remanrau foras 84

A pena e a torment. $\quad 85$

33 Spiritus sancs aor 86

E prec lo de s'amor 87

Fasam cosolament. $\quad 88$

$34 \mathrm{El}$ es vers consolaire, 89

Qu'es del fil e del paire 90

Ambedos egalment. $\quad 9 \mathrm{I}$

13a Meyer deffiet; b Meyer baiset. 15a folort: Batutz e malmenatz vgl. I6c, welches in der Handschrift fehlt. $17 \mathrm{c}$ el von Meyer ergänat. 2Ib Lo pair de sus; 2 Ic Que lor fasa p. $22 \mathrm{c}$ Se autre fa m. 23c Li mes. quero mescladament $24 \mathrm{~h}$ gostar mit überschriebenen $\mathrm{u}$; goustar Meyer.

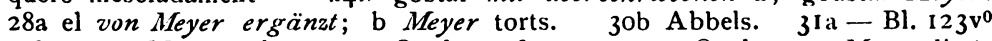
3I estan Meyer, seis. 33c Quel me fasa c. 34a Quel v. c., Meyer liest: Aquel. 
$35 \mathrm{E}$ aor trinitat $\quad 92$

$\mathrm{E}$ una deïtat 93

Aisi perfectament. $\quad 94$

36 Aiso es ma creensa 95

Secun ma conoisensa 96

A trasaiadament

97

37 Quant Deus per mi fo morts 98

De m'anma e de mo cors 99

Li fas bo garniment. 100

$3^{8}$ Per la golat me ren, ror

Mas mas juntas t'esten, 102

E! bos Sener, tum pren! 103

39 Dot me per totas fes, 105

Que ja mai mala res 106

No i aia raisnament. $\quad 107$

40 E gurpis lo diable, 108

L'enjenios el mudable, I09

$E$ cant a lui apen. I IO

4I E seni'n mo carah III

Aquel que tu m'as faih 112

Fn aquel covinent. II 3

In nomine patris et filii et spiritus sancti amen.

II.

I Damrideus receb me, 114

Que eume ret a te 115

F. cofes e penent 116

2 Des pecaz que ai faïz

E diz e cosiratz $\quad 117$

E del(s) mesprendement 118

3 De l'ora que fui naz i 19

E e fons babtizats 120

Tro en est jorn present. 121

4 Tu est vers prestre e Deus, 122
Eu pecaire soi teus 123

Cofes membradrament, $\quad 124$

5 Pos fui naz de ma maire,

Comensei mal a faire 126

Contra teu mandament. 127

6 E eul fis e eul dis. 128-9

El diables l'escris I 30

Pel meu acusament. I 13

7 So qu'el mes en escrit, 132

Tot l'as tu tost delit 133

$\mathrm{E}$ tornat a neient. $\quad 134$

8 De ma longa malesa, 135

De ma laia oreesa $\quad{ }_{136}$

Te fas cofesament, $\quad \mathbf{3 7}$

9 De tantas guisas laias 138

Ai orreesas faitas. $\quad 139$

Las, pecaire dolent! $\quad 140$

Io De mos pecaz tant orz Ei eu dol e mo cor I4I

$E$ ira e marriment. $\quad \mathbf{1 4 2}$

II Eu mesis m'en acus, 143

Que anc om no'n fes plus, 144

$\mathrm{Ni}$ ac mos cors entent. 145

12 E d'aiso, so cofes, 146 Mensungas dic ades, 147

E ment mo sagrament. 148

13 Que jur ti e ta maire, 149

$\mathrm{E}$ tot lo teu afaire 150

D'aso don sei que ment. I5I

14 Engans e laironias, 152

Traïsos e bausias $\quad 153$

Ei fait mon esient. $\quad 154$

15 E veïr e hauvir, $\quad 155$

E baiar e sentir $\quad 156$

Ai mespres mot soent. 157

$37 \mathrm{c}$ bo von Meyer ergänzt.

Queu tem do ses tot calumnament. Meyer las: E s'e ran mo c., was unverständlich bleibt, und auch paläographisch unzulässig ist, vgl. ra in carah, in delivrament 70, mostral 53 etc.

Ia Deus r. me; Ib so Hs. Q'eu Meyer, der dann auch noch ändert: eu m'ret; Ic E fehlt. 2a qu'ai Meyer; 2c del Meyer, der Schreiber wollte wohl eigentlich mesprendements schreiben, wie er auch Ic erst penens schrieb. 4 a Tu si est v. pr. e (et Mejer) vers D.; 4 b E eu p. qui s. t.; 4c A tim c. m. 5a Pos Bartsch, Mos Hs. 6a E eu (scheint in tu gebessert zu sein) lo f. e eu lo d. 7a qu'el escris; $7 \mathrm{~b} E$ tu delis; $7 \mathrm{c} \mathrm{E}$ torna. $8 \mathrm{~b}$ laia malesa, zum Ersatz von male ist aber ore übergeschrieben, also oresa überliefert, oreesa Meyer. 9b oreezas Meyer. Ioa fehlt in der Hs.; 10 en. IIc so scors, Meyer so cors. $14 \mathrm{~b}$ traïsios Meyer. $15 \mathrm{a} \mathrm{E}$. v. Hh. $=(i m$ sehen $u n d$ im hören), Mejer et. I5b ee. 15C Ei mit übergeschriebenem a; soet, Meyer soe $[\mathrm{n}] \mathrm{t}$. 
I6 Se re vei que m'agrat, 158

Ei mala volontat, $\quad 159$

E d'aiso sim repen. $\quad 160$

I7 De tot aisom penet I6I

$E$ a merset m'en ret 162

Qu'en fasas to talent. 163

I 8 Fait ei pecatz mortals, I 64

Dignes soi de toz mals, 165

Se ti pietat no'n pren. $\quad 166$

19 Des mals e des deleits 167

$\mathrm{Me}(\mathrm{n})$ feri en est peits 169

Mia colpa dizen. $\quad 170$

20 Mia colpa'n dic eu, I7I

E si la'n fas. vas Deu 172

E ma colpa'n enten. $\quad$ I73

2 I Jesus bos, Jesus douz, 174

Mercet quer per tots noms 175

E ver castiament. $\quad$ I 75

22 Mercet quer per ta maire 177

A ti e a to paire $\quad 178$

Del mal contenement $\quad 179$

23 Que ei ades tegut, 180

Et ei vas vos agut $18 \mathrm{I}$

Ades e mo jovent. $\quad 182$

24 Fais me merce tuh tres, 183

Que al diable pes 184

Del meu melurament! $\quad 185$

25 Jesu, per ta bontat $\quad 186$ Garam de lait pecat, 187

De blasme e d'auniment! 188

26 E d'aquels que ei fait, 189

D'aquels i a asat, $\quad 190$

Fai me perdonament! I9I

27 Bos Deus, paubr'es ma fes 192-3

$E$ res estre merces 197.8

Nom mena a salvament. 199
28 Et aquela merces 200

Que gueri ja los tres 201

De la fornaz ardent 202

29 El'estena, set plats, 203

Las flamas des pecatz 204

E mo cor esmament, 205

30 Em salve em govern 206

De las penas d'efern 207

$E$ de tot mal torment. 208

3I Deus gardam de vergona 209

E de m'autra besona 210

Fai m'en socorrement! 2II

32 Deus de mos enamics 212

Des paubres e des rics, 213

Fai m'en mantenement! 214

33 E tots cels que m'atano 2 I5

Que bem volo ni m'amo 216

Gardam d'afolament! 217

34 Prec te per ta pietat 218

E per ta voluntat, 218

Set plaz privadament, 220

35 Quem cosentas amor $22 \mathrm{I}$

Quem sal e quem conort 222

Mo cors membradament, 223

36 E quem fasas saber 224 Ma fi, set plaz, a te 225

O quer fortismament. 226

37 E prec te per tas plagas, 227 Que de mi merce aias 228

Al teu aveniment, $\quad 229$

38 E prec te per ta crost 230

$E$ per ta sancta vots $22 \mathrm{I}$

Que disit umilment, $\quad 232$

39 Quant abellest Eli 233

El cap tenguist ecli 234

Al paire omnipoten, 235

$16 \mathrm{a}$ Se re $\left(\mathrm{Bl} .123 \mathrm{v}^{\circ}\right)$ vei. $17 \mathrm{c}$ talant mit übergeschriebenem e. 18 apecat. I9a deleit folgt: I68 Qu'ai fait (Meyer fai) pes teus despeis). I9c peit. $2 \mathrm{Ia}$ Meyer Jhesus. $2 \mathrm{Ib}$ tot. $22 \mathrm{~b}$ E (Meyer [A) ti a ta maire (ein ähnlicher Fehler ist $8 b$ von Schreiber selbst gebessert). $22 \mathrm{c}$ De. 23 a ades fehlt, tengut Meyer; 23b \& Meyer e, vas vos fehlt. 26b D'aquel, Meyer D'aque][s]. 27a-c Bos Deus, so.ves, 193 Paubres m'a fes 194 Paucs es mos bes $195 \mathrm{El}$ mas es gràn (Meyer) El mal gran es) 196 Que e mi es; $197 \mathrm{E}$ nula res 198 Estre merces 199 Menar nom (Meyer non) pot a salvament. Der Meyer'sche Text ist sonst abgesehen von der Zeileneinteilung und der falschen Worttrennung in Z. 193 von der Hs. überliefert. 28a Et fehlt; 28b gueri stres, Meyer gueris [los] tres. 29a El' fehlt. 29a pecat, Meyer del pecat. 29c, vgl. I 3c. 30a Cel me s.; 3oc; de von Meyer ergänzt. 32a Deus gardam de m. e. $33^{a}$ De. $34^{a}$ pieta, Meyer pieta[t]. 35b Que, Meyer Que[m'], conorts. $36 \mathrm{~b}$ plaz t'o quer (Schon der vokalisch unzulässige Reim nötigt zu einer Anderung). $\quad 36 \mathrm{c} O$ quer fehlt. 
40 Quem tolas de senestre 236

Em metas al las dextre 237

Al teu sanc jutgament. 238

41 E pecats criminals 239

$\mathrm{Ni}$ negus autre mals 240

Nom si'a damnament. 24I

42 Perdonam per ta mort 242

Ta ira don a tort 243

Non fas emendament! 244

43 Per ta resurrexio 245

Aunis ma oraso: $\quad 246$

Deus meus amen! 247
44 E pel teu nom mirable 248

Defen me de diable, 249

D'efern e del torment, 250

45 E met m'e paradis $25 \mathrm{I}$

On om no velesis 252

$\mathrm{Ni}$ no mor ni no ment, 253

46 Mi e mos bevolents 254

E mos propris parenz 255

Totas tas autras autras gens! 256

(Que eu dic pater noster pel seu entendemen.)

$4 \mathrm{ra}=\mathrm{Bl} . \mathrm{I}_{2} \mathrm{r}^{\mathrm{O}}$ pecat criminal; $4 \mathrm{Ib}$ mal. $\quad 4 \mathrm{Ic}$ Meyer sia. $\quad 42 \mathrm{~b} \mathrm{mal}$. $42 \mathrm{~b}$ ai t.; $42 \mathrm{c}$ Que non posc far e. $43 \mathrm{a}$ E per; $43 \mathrm{~b}$ E s'auuis. $45^{\mathrm{b}} \mathrm{Mejer}$ novelesis (ce verbe, l'opposé de velhesir n'est pas dans Raynouard). 46 bevolent.

E. Stengel. 